\title{
Nested PCR detection of Plasmodium malariae from microscopy confirmed $P$. falciparum samples in endemic area of NE India
}

\author{
Sunil Dhiman ${ }^{1}$, Diganta Goswami ${ }^{1}$, Dinesh Kumar ${ }^{1}$, Bipul Rabha ${ }^{1}$, Dhirendra Kumar Sharma ${ }^{2}$, Rakesh Kumar \\ Bhola $^{2}$, Indra Baruah ${ }^{1}$ and Vijay Veer ${ }^{1}$
}

${ }^{1}$ Department of Medical Entomology, Defence Research Laboratory, Tezpur, Assam, India;

${ }^{2}$ Department of Zoology, Gauhati University, Guwahati, Assam, India

\begin{abstract}
The present study evaluates the performance of OptiMAL-IT ${ }^{\circledR}$ test and nested PCR assay in detection of malaria parasites. A total of 76 randomly selected blood samples collected from two malaria endemic areas were tested for malaria parasites using microscopy and OptiMAL-IT ${ }^{\circledR}$ test in the field. PCR assays were performed in the laboratory using DNA extracted from blood spots of the same samples collected on the $\mathrm{FTA}^{\mathrm{TM}}$ classic cards. Of the total of 61 field confirmed malaria positive samples, only 58 (95\%) were detected positive using microscopy in the laboratory. Sensitivity, specificity, positive predictive value, negative predictive value and false discovery rate of OptiMal-IT ${ }^{\circledR}$ in comparison to the microscopy were $93 \%, 83 \%, 95 \%, 79 \%$ and $5 \%$, respectively. On the other hand, the sensitivity and specificity of PCR assay were $97 \%$ and $100 \%$, respectively, whereas positive predictive value, negative predictive value and false discovery rate were $100 \%, 90 \%$ and $0 \%$, respectively. The overall performance of OptiMal-IT ${ }^{\circledR}$ and PCR assays for malaria diagnosis was $76 \%$ and $97 \%$, respectively. PCR assay enabled the identification of infection with Plasmodium malariae Laveran, 1881 in four samples misidentified by microscopy and Plasmodium-specific antigen (PAN) identified by the OptiMAL-IT ${ }^{\circledR}$ test. In addition to the standard methods, such PCR assay could be useful to obtain the real incidence of each malaria parasite species for epidemiological perspectives.
\end{abstract}

Keywords: malaria, diagnostics, blood samples, OptiMAL-IT ${ }^{\circledR}$, sensitivity, specificity, Assam

Malaria is a complex and evolved infectious human disease that is endemic in north-eastern states of India, where malaria control relies on early active and passive case detection and prompt treatment through malaria camps and village level health workers (Dhiman et al. 2011). Since malaria associated with fever and other symptoms is the most common diagnosis in rural settings, the incorrect diagnosis may have severe public health implications (Anash et al. 2010). A considerable proportion of patients treated for malaria actually do not have malaria (Prasad 2009). Furthermore, missed out true malaria cases may act as epicentres for disease transmission at a local level. Resistance to anti-malarials, decay in health infrastructure, population movement and socio-political unrest are contributing factors to the spread of malaria (Gardner et al. 2002). Accurate diagnosis of malaria cases could be useful in lowering such conjectures and ultimately reducing malaria burden in endemic settings.

Until recently, microscopic examination of blood smears has been a standard method for malaria diagnosis by convenience (Anash et al. 2010). Microscopy, however, requires well trained technical staff and it is also rather labour intensive and time consuming (Maltha et al. 2010). Hand-held non-microscopic immunochromatographic rapid diagnostic tests (RDT's) are simple, sensitive and specific, and good results have been achieved with them in various endemic regions. Since their introduction during the late 1990's, RDT's have undergone many changes and currently three-band RDT's for detecting both Plasmodium falciparum Welch, 1897 specific antigen and Plasmodium-specific antigen (PAN-specific) have been developed (Moody 2002).

The performance of the RDT's in the diagnosis of malaria has been validated throughout the malaria endemic regions (Moody 2002, Anash et al. 2010), however, the RDT's were unable to identify mixed infections, owing to the common PAN-specific antibody capture band, when compared to the polymerase chain reaction (PCR) assay. In addition, the RDT's have not been found suitable to diagnose malaria where parasite counts were as low as $<50$ parasite/ $\mu$ l (Iqbal et al. 1999).

The pLDH based OptiMAL-IT ${ }^{\circledR}$ has some limitations, but as on today, it has been proved to be one of the best RDT available for on spot detection of malaria parasite 
(Moody 2002). In the recent years, PCR assay has been proved to be a sensitive method to diagnose the malaria parasite at species level and in mixed infections, and is expected to detect parasite at very low level (Iqbal et al. 1999, Scopel et al. 2004).

In the north-east India, $P$. falciparum malaria, in particular, emerges with high degree of antimalarial drug resistance and therefore treatment regime differs from that used for the other malaria parasite species. The correct malaria treatment relies on the proper diagnosis of malaria parasite and following the recommended chemotherapy. In the field settings in India, where malaria diagnosis at large is carried out using microscopy, incorrect diagnosis is common and leads to progression of uncomplicated malaria to severe disease (Prasad 2009). Correct treatment would interrupt the indigenous transmission and minimizes the risk of selection and spread of drug resistance to the other areas.

Plasmodium malariae Laveran, 1881 is still uncommon in India and only a few systematic studies have reported it in the country. In the northeastern region, only two studies have reported the presence of $P$. malariae malaria (Dev 2000, Mohapatra et al. 2008), however, only the latter study has evidenced the persistence transmission of $P$. malariae in the region. Molecular methods have the merit of differentiating morphologically similar Plasmodium species. The present study was carried out to evaluate the usefulness of PCR assay in malaria diagnosis, detecting the mixed infection and identifying the malaria parasite species, which have been, otherwise, missed out.

\section{MATERIAL AND METHODS}

\section{Study area and sample collection}

With the help of local medical authority, Defence Research Laboratory organises malaria camps for civil population inhabiting the surrounding areas of defence cantonments. All the malaria positive cases are given antimalarial treatment as prescribed by National Vector Borne Disease Control Programme (NVBDCP). The present samples were collected from patients that reported for malaria testing in the malaria camps during the year 2011 from two different malaria endemic clinical sets, Missamari $\left(92^{\circ} 35^{\prime} 34^{\prime} \mathrm{E} ; 26^{\circ} 48^{\prime} 11^{\prime \prime N}\right)$ and Hozai $\left(92^{\circ} 50^{\prime} 47^{\prime \prime} \mathrm{E}\right.$; $\left.26^{\circ} 00^{\prime} 14^{\prime \prime} \mathrm{N}\right)$, both in Assam, India.

These two areas report $P$. falciparum and $P$. vivax Grassi et Feletti, 1890 cases and no evidence of other human malaria parasite infection are available in these areas. Missamari is a foothill area dominated by various ethnic tribes, whereas Hozai is relatively plain area inhabited by mixed (tribal and non-tribal) population. Bodo and Rabha are main ethnic tribes in Missamari, whereas Karbi, Dimasa and Bodo tribes inhabit Hozai area. We conducted a double blind study to compare the performance of smear reading using microscopy and PCR, while masked to the RDT results in detecting possible malaria parasite species other than the two species encountered frequently.

A total of 61 (37 from Missamari and 24 from Hozai) microscopically confirmed malaria samples and 15 confirmed negative randomly-selected samples were used for the present study.
The entire samples were parallely checked using OptiMAL-IT ${ }^{\circledR}$ kits (Diamed AG; Lot No. 46110.72.01) in the field and at the same time, 2-3 drops of blood were collected on the FTA ${ }^{\mathrm{TM}}$ classic cards (Whatman, Sweden) for PCR assays. These randomly selected 76 samples were re-examined by another senior technician in the laboratory using light microscopy. In case of discrepancies, the results obtained during re-examination using microscopy were recorded as true and used for the present study. The study was approved by the Ethical Review Committee of L.G.B. Regional Institute of Mental Health, Tezpur and informed verbal consents were obtained from the patients.

\section{DNA preparation and nested PCR assay}

Parasite DNA was extracted using Qiagen kit and following the standard FTA ${ }^{\mathrm{TM}}$ manufacturer procedure. Plasmodium species identification was done by nested PCR amplification (Bio-Rad S1000) of the small subunit of the ribosomal ribonucleic acid (18S rRNA) genes using the primers for four human-infecting malaria parasite species excluding $P$. knowlesi, Knowles et Gupta, 1931. PCRs were performed essentially with the primers described elsewhere (Snounou et al. 1993, Johnston et al. 2006). The cycling conditions were similar to Johnston et al. (2006), except for increase in reaction cycles to 35 in the first round and decrease in annealing time to one minute in the nested step for $P$. falciparum, $P$. vivax and $P$. malariae. Genomic DNA isolated from the blood of one of the investigators with no malaria history was used as negative control in the PCR assays. The amplified PCR products were resolved by $2 \%$ agarose gel electrophoresis and viewed using UV transilluminator (Syngene G-box).

\section{Data analysis}

The sensitivity and specificity of the OptiMAL- IT ${ }^{\circledR}$ and PCR assay for the detection of malaria infection were calculated by using microscopy as reference standard. Positive and negative predictive values (NPV and PPV) referred to the probability that the disease is present and absent when the test is positive and negative, respectively. Positive and negative likelihood ratios (LR) were the probability of a test result with the disease. False discovery rate (FDR) was the proportion of false-positive results out of all positive tests. Overall performance (\%) of OptiMAL$\mathrm{IT}^{\circledR}$ and PCR assay in diagnosing malaria was calculated using Youden method (J) (Youden 1959).

\section{RESULTS}

Out of a total of 61 microscopy-confirmed malaria positive blood smears in the field, only $58(95 \%)$ could be detected positive using microscopy in the laboratory. However, the difference between the field and laboratory microscopy results was not significant $[\mathrm{p}$-value $(\mathrm{p})$ $=0.69$; chi-square $\left(\chi^{2}\right)=0.15$; relative risk $(\mathrm{RR})=1.128$; $95 \%$ confidence interval $(95 \% \mathrm{CI})=0.7463-1.704]$. Of these 58 blood smears, $53(91 \%)$ were positive for Plasmodium falciparum and $5(9 \%)$ were positive for $P$. falciparum and $P$. vivax mixed infection (Table 1). The OptiMal-IT ${ }^{\circledR}$ identified $P$. falciparum in $49(86 \%)$ and PAN in $8(14 \%)$ individuals in the field.

Four P. falciparum infections confirmed by microscopy were not detected positive by OptiMal-IT ${ }^{\circledR}$ test. On the other hand, OptiMal-IT ${ }^{\circledR}$ had detected three P. falciparum infections which were not detected by microscopy in the 
Table 1. Comparison of OptiMAL-IT ${ }^{\circledR}$, nested PCR assay and microscopy for detection of Plasmodium infection in 76 samples.

\begin{tabular}{|c|c|c|c|}
\hline Plasmodium species & Microscopy & OptiMAL-IT $^{\circledR}$ & Nested PCR \\
\hline P. falciparum & 53 & 49 & 48 \\
\hline P. malariae & - & - & 1 \\
\hline P. falciparum/P. vivax & 5 & - & 4 \\
\hline P. falciparum/P. malariae & - & - & 3 \\
\hline PAN & - & 8 & - \\
\hline Negative & 18 & 19 & 20 \\
\hline
\end{tabular}

PAN - Plasmodium-specific antigen.

Table 2. Overall performance of OptiMAL-IT ${ }^{\circledR}$ and nested PCR in comparison to microscopy.

\begin{tabular}{lcccccccccc}
\hline $\begin{array}{l}\text { Diagnosis } \\
\text { method }\end{array}$ & M $\begin{array}{l}\text { Sensi- } \\
\text { tivity }\end{array}$ & $\begin{array}{c}\text { Specifi- } \\
\text { city }\end{array}$ & PPV & NPV & LR+ & LR- FDR & J \\
\hline OptiMAL-IT $^{\circledR}$ & 57 & 93.1 & 83.3 & 94.7 & 78.9 & 5.6 & 0.1 & 5.3 & 0.8 \\
Nested PCR & 56 & 96.6 & 100 & 100 & 90 & 1 & 0 & 0 & 1 \\
\hline
\end{tabular}

$\mathrm{M}$ - diagnosed positive; PPV - positive predictive value; NPV - negative predictive value; LR (+/-) - likelihood ratio (positive/negative); FDR - false discovery rate; $\mathrm{J}$ - Youden coefficient.

laboratory. Sensitivity and specificity of OptiMal-IT ${ }^{\circledR}$ as compared to microscopy was $93 \%$ and $83 \%$, respectively. PPV, NPV and FDR for OptiMal-IT ${ }^{\circledR}$ were $95 \%, 79 \%$ and $5 \%$, respectively (Table 2). The overall performance of OptiMal-IT ${ }^{\circledR}$ recorded for malaria diagnosis in the present study was $76 \%$.

PCR assay detected P. falciparum in 48 (86\%) blood samples, P. falciparum and P. vivax mixed in $4(7 \%)$, $P$. falciparum and $P$. malariae mixed in $3(5 \%)$ and P. malariae in one (2\%) sample. Four P. falciparum and $P$. vivax mixed infections detected by microscopy were detected correctly by PCR. However, in another case, one microscopy detected $P$. falciparum and $P$. vivax mixed infection was identified as $P$. falciparum and P. malariae mixed infection by PCR. One $P$. falciparum was identified as P. malariae and two P. falciparum were identified as $P$. falciparum and $P$. malariae mixed infections by PCR.

Three microscopy-detected negative samples were confirmed to be negative by PCR assay, whereas these samples were detected as positive by OptiMAL-IT ${ }^{\circledR}$. Two microscopy-positive samples could not be detected positive either by OptiMAL-IT ${ }^{\circledR}$ or PCR. The sensitivity and specificity of PCR assay in detecting malaria parasite was $97 \%$ and $100 \%$, respectively, whereas PPV, NPV and FDR were $100 \%, 90 \%$ and $0 \%$, respectively. The overall performance of PCR assays was $97 \%$.

No P. malariae infection was diagnosed using microscopy in the present study. However, nested PCR detected three $P$. falciparum and $P$. malariae mixed infections and one $P$. malariae monoinfection. The nested PCR ampilicons of $P$. malariae infections were visualised at $144 \mathrm{bp}$ diagnostic band (Fig. 1), whereas P. falciparum and

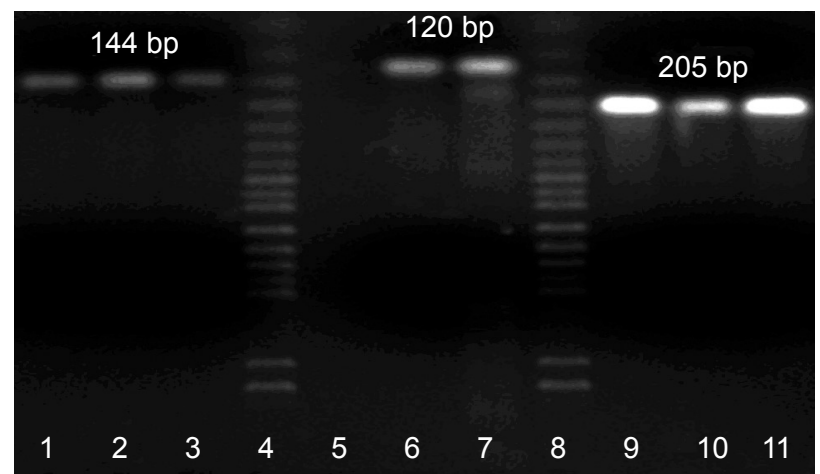

Fig. 1. Detection of Plasmodium species amplification in nested PCR. Lanes 1-3: Plasmodium malariae (144 bp); lanes 6, 7: P. vivax (120 bp); lanes 9-11: P. falciparum (205 bp); lanes 4 and 8: standard ladder Sigma (50 bp); lane 5: negative control.

P. vivax were resolved at 205 and 120 bp positions, respectively. Plasmodium malariae infections were identified from three males and one female patients of different age groups (11-55 years) belonging to different tribes.

\section{DISCUSSION}

The performance of OptiMAL-IT ${ }^{\circledR}$ test kit and nested PCR assay in detecting the malaria parasite was evaluated using randomly selected samples $(n=76)$ from two highly endemic areas of Assam. Out of 61 confirmed samples, $95 \%$ were identified malaria positive in the second round microscopy. In the present study, microscopy detected one $P$. vivax and two $P$. falciparum cases, which were later identified correctly as P. malariae by PCR.

Microscopic examination has been the diagnostic method of choice in malaria epidemiologic studies and diagnosis. The method is simple, does not require special facilities and enables differentiation among the four well known species of human malaria parasite in most cases (Maltha et al. 2010). Microscopy has limitation that even a skilled person can evaluate only limited number of blood smears per day under field conditions (Gardner et al. 2002). Furthermore, in case of low level of parasitemia and mixed parasite infection, microscopy can sometimes be misleading in identification of malaria parasite species.

The sensitivity and specificity of OptiMAL-IT ${ }^{\circledR}$ was 93\% and $83 \%$. OptiMal-IT ${ }^{\circledR}$ detects P. falciparum and PAN-specific antigen for Plasmodium species. However, four microscopy-confirmed P. falciparum cases were detected negative by OptiMAL-IT ${ }^{\circledR}$. The sensitivity of OptiMAL-IT ${ }^{\circledR}$ has been found low with the lower level of parasitemia or presence of blocking antibodies in the blood. OptiMAL-IT ${ }^{\circledR}$ test also detected three false P. falciparum cases, probably due to the presence of pLDH antigen, which has been found to remain in the patient's blood even after initiation of the treatment with antimalarials (Iqbal et al. 1999). The reading beyond the reading time may increase the number of positive PAN-pLDH 
lines, apparently explaining for the false-positive cases observed in the tests (Maltha et al. 2010). In the PCR assay, the sensitivity and specificity were $97 \%$ and $100 \%$, respectively.

Similar to the present study, the PCR assays have been proved useful in detecting mixed Plasmodium infections in various malaria endemic regions (Kimura et al. 1995, Cox-Singh et al. 2008). The PCR assays in the present study were found better than the microscopy in detecting Plasmodium species, which were misidentified by microscopy. In mixed infections, most of the time one Plasmodium species either dominates or inhibits the other Plasmodium species present inside the same red cell population (Kimura et al. 1995).

Two microscopy positive $P$. falciparum samples were detected negative in PCR assay. These two patients were under chloroquine treatment for last 2-3 days and the smears contained only few live parasites. Most of the $P$. falciparum parasites in these smears were either moribund with irregular shape or dead. It has been shown that the DNA extracted from dead parasites did not show positive amplification in PCR assay (Tham et al. 1999). The DNA extracted from these two samples was either unfit for amplification due to poor quality or probably lost during the storage and handling.

In two $P$. falciparum cases, $P$. malariae was identified along with $P$. falciparum as mixed infections, whereas one $P$. falciparum and $P$. vivax mixed infection detected by microscopy was identified as $P$. falciparum and $P$. $m a-$ lariae mixed infection by PCR. Many malaria cases in north-eastern region of India appear with $P$. falciparum and $P$. vivax mixed infection, but only a few cases of $P$. falciparum with $P$. malariae and $P$. falciparum with $P$. vivax mixed infection have been repoted (Mohapatra et al. 2008). Mixed infection with two or more Plasmodium species is largely underestimated when microscopy is used in malaria detection instead of molecular-based diagnosis (Cox-Singh et al. 2008).

Plasmodium malariae is not very common in this region and has been reported only in two states of northeastern India (Dev 2000, Mohapatra et al. 2008), and its prevalence in other parts of the region is unknown (Mohapatra et al. 2008). It appears that due to the large number of samples and preconceived notion of diagnosing fre- quently encountered $P$. falciparum and $P$. vivax malaria parasites, the examiner might have missed the $P$. malariae infection in the present study. In microscopy, $P$. malariae may be confused with other Plasmodium species due to the similarity of the growth stages (Scopel et al. 2004). Many studies have revealed that $P$. knowlesi is often misdiagnosed as P. malariae in South East Asian countries. Infections with $P$. knowlesi are thought to be potentially more serious and even life-threatening if wrongly treated or remain untreated (Cox-Singh et al. 2008).

Use of antimalarials for malaria treatment in any area largely depends on the type of malaria species present in that particular area. Delayed or wrong diagnosis of malaria parasites increases the risk of complicated disease and relapses, which may be fatal in many cases. Further, due to negative diagnosis, untreated patients may be carriers of malaria parasites and act as epicentres for other non-infected individuals. Plasmodium malariae parasite is important from an epidemiological perspective, unlike other malaria parasites, as it can sustain at very low infection rates within the human hosts for many years. This parasite is capable of infecting the mosquito vectors even at very low parasitemia, thus facilitating transmission round the year (Mohapatra et al. 2008).

The current study region falls in malaria zone 2 where, although falciparum malaria is dominant, it typically cocirculates and occurs together with the non-falciparum malaria (Murray and Bennett 2009). OptiMAL-IT ${ }^{\circledR}$ test in such settings in not much useful in detecting the mixed infections to the species level. Therefore, the nested PCR detection of malaria parasites complemented with the other existing methods is useful to timely obtain the incidence of each malaria parasite species for specific treatment and epidemiological follow up, despite the possibility of rare false positive cases. The present study emphasizes that $P$. malariae may be more prevalent in the region than thought previously. PCR assays employed here may not be used routinely due to various constraints, but can be used at least at primary health centre level for re-evaluation of existing methods.

Acknowledgments. Authors are thankful to the local health authorities of Assam State for providing samples and logistic support during the study.

\section{REFERENCES}

Ansah E.K., Narh-Bana S., Epokor M., Kanpigbiam S., Quartey A.A., Gyapong J., Whitty C.J.M. 2010: Rapid testing for malaria in settings where microscopy is available and peripheral clinics where only presumptive treatment is available: a randomised controlled trial in Ghana. Br. Med. J. 340: c930.

Cox-Singh J., Davis T.M., Lee K.S., Shamsul S.S., Matusop A., Ratnam S., Rahman H.A., Conway D.J., Singh B. 2008: Plasmodium knowlesi malaria in humans is widely distributed and potentially life threatening. Clin. Infect. Dis. 46: 165-171.
Dev V. 2000: Plasmodium malariae - a case of quartan malaria in Assam. J. Commun. Dis. 32: 149-151.

Dhiman S., Gopalakrishnan R., Goswami D., Rabha B., BaruAH I., Singh L. 2011: Malaria incidence among paramilitary personnel in an endemic area in northeastern India. Ind. J. Med. Res. 133: 665-669.

Gardner M.J., Hall N., Fung E., White O., Berriman M., Hyman R.W., Carlton J.M., Pain A., Nelson K.E., Bowman S., Paulsen I.T., James K., Eisen J.A., Rutherford K., 
Salzberg S.L., Craig A., Kyes S., Chan M.S., Nene V., Shallom S.J., Suh B., Peterson J., Angiuoli S., Pertea M., Allen J., Selengut J., Haft D., Mather M.W., Vaidya A.B., Martin D.M., Fairlamb A.H., Fraunholz M.J., Roos D.S., Ralph S.A., McFadden G.I., Cummings L.M., Subramanian G.M., Mungall C., Venter J.C., Carucci D.J., Hoffman S.L., Newbold C., Davis R.W., Fraser C.M., Barrell B. 2002: Genome sequence of the human malaria parasite Plasmodium falciparum. Nature 419: 498-511.

Iqbal J., Sher A., Hira P.R., Al-Owaish R. 1999: Comparison of the OptiMAL test with PCR for diagnosis of malaria in immigrants. J. Clin. Microbiol. 37: 3644-3646.

Johnston P.S., Pieniazek N.J., Xayavong M.V., Slemenda S.B., Wilkins P.P., Silva A.J. 2006: PCR as confirmatory technique for laboratory diagnosis of malaria. J. Clin. Microbiol. 44: 1078-1089.

Kimura M., Miyake H., Kim H.S., Tanabe M., Arai M., Kawai S., Yamane A., Wataya Y. 1995: Species-specific PCR detection of malaria parasites by microtiter plate hybridization: clinical study with malaria patients. J. Clin. Microbiol. 33: $2342-$ 2346.

Maltha J., Gillet P., Bottieau E., Cnops L., Esbroeck M.V., JACOBS J. 2010: Evaluation of a rapid diagnostic test (CareStart ${ }^{\mathrm{TM}}$ Malaria HRP-2/pLDH (Pf/pan) Combo Test) for the diagnosis of malaria in a reference setting. Malar. J. 9: 171.

Mohapatra P.K., Prakash A., Bhattacharyya D.R., Goswami B.K., Аhmed A., Sarmah B., Mahanta J. 2008: Detection

Received 14 November 2012 and molecular confirmation of a focus of Plasmodium malariae in Arunachal Pradesh, India. Ind. J. Med. Res. 128: 52-56.

Moody A. 2002: Rapid diagnostic tests for malaria parasites. Clin. Microbiol. Rev. 15: 66-78.

Murray C.K., Bennett J.W. 2009: Rapid diagnosis of malaria. Interdiscip. Perspect. Infect. Dis. ID 415953.

PrASAD H. 2009: Evaluation of malaria control programme in three selected districts of Assam, India. J. Vector Borne Dis. 46: $280-287$.

Scopel K.K., Fontes C.J., Nunes A.C., Horta M.F., Braga E.M. 2004: High prevalence of Plasmodium malariae infections in a Brazilian Amazon endemic area (Apiacas - Mato Grosso State) as detected by polymerase chain reaction. Acta Trop. 90: 61-64.

Snounou G., Viriyakosol S., Jarra W., Thaithong S., Brown K.N. 1993: Identification of the four human malarial species in field samples by the polymerase chain reaction and detection of a high prevalence of mixed infections. Mol. Biochem. Parasitol. 58: 283-292.

Tham J.M., Lee H.S., Tan T.M.C., Ting R.C.Y., Kara U.A.K. 1999: Detection and species determination of malaria parasites by PCR: comparison with microscopy and with ParaSight$\mathrm{F}$ and ICT malaria Pf tests in a clinical environment. J. Clin. Microbiol. 37: 1269-1273.

Youden W.J. 1959: Graphical diagnosis of interlaboratory test results. Ind. Qual. Cont. 15: 24-28.

Accepted 7 May 2013 